\title{
NUEVOS REGISTROS DE ECTOPARASITOS (INSECTA: DIPTERA) DE MURCIELAGOS (CHIROPTERA) DEL NORDESTE ARGENTINO
}

\author{
Elena B. Oscherov(1); Fabricio M. Idoeta ${ }^{(2)}$ y Alicia M. F. Milano(1)
}

RESLMEN: Los murciélagos, por sus características ecológicas particulares, se asocian con una fauna de artrópodos en su mayoría específica. El objetivo de este trabajo fue ampliar el conocimiento sobre dípteros ectoparásitos de murciélagos en la provincia de Corrientes. Los murciélagos se capturaron durante diciembre de 2008 a noviembre de 2009 en Isla Apipé Grande, Puerto Valle y Rincón Santa María. Los hospedadores se obtuvieron por captura manual y con redes de niebla, expuestas entre las 19 y las 5 hs, tres noches en cada localidad. Los ectoparásitos se conservaron en una solución de alcohol etílico y glicerina y en laboratorio se procedió a la identificación, utilizando claves específicas. Las especies que presentaron ectoparásitos fueron Sturnira lilium (Phyllostomidae), Eptesicus furinalis y Myotis nigricans (Vespertilionidae). Los dípteros identificados fueron Megistopoda proxima (Streblidae) Basilia plaumanni y Basilia carteri (Nycteribiidae). Se citan por primera vez B. plaumanni y $M$. proxima para la provincia de Corrientes y se amplía la distribución de $B$. carteri a la eco-región Delta e Islas del Paraná.

ABSTRACT: Because of their particular ecological characteristics, bats are associated with an arthropod fauna that is mostly specific. The aim of this work was to widen the knowledge about bats diptera ectoparasites in the Province of Corrientes. Bats were captured in Isla Apipe Grande, Puerto Valle and Rincon Santa Maria during the months of December 2008 to November 2009. Hosts were manually captured with fog nets exposed during three nights from $7 \mathrm{pm}$ to 5 am in each town. Ectoparasites were preserved in a glycerin and ethanol solution and afterward identified in the lab using specific keys. Among them, the species who presented ectoparasites were Sturnira lilium (Phyllostomidae), Eptesicus furinalis and Myotis nigricans (Vespertilionidae). Identified dipteria were Megistopoda proxima (Streblidae) Basilia plaumanni and Basilia carteri (Nycteribiidae). B. plaumanni y M. proxima are first cited in the Province of Corrientes while $B$. carteri widens its distribution to the Delta eco-region and the Parana islands.

Palabras claves: Streblidae, Nycteribiidae, parásitos, quirópteros, Phyllostomidae, Vespertilionidae, Corrientes, Argentina

Key words: Streblidae, Nycteribiidae, parasites, bats, Phyllostomidae, Vespertilionidae, Corrientes, Argentina

\section{INTRODUCCIÓN}

En la provincia de Corrientes están representadas cinco de las 15 eco-regiones continentales de Argentina: Chaco Húmedo, Esteros del Iberá, Deltas e Islas del Paraná,

(1) Biología de los Parásitos, Facultad de Ciencias Exactas y Naturales y Agrimensura, UNNE. Av. Libertad 5470 (3400) Corrientes, Argentina. E-mail: ebosche@exa.unne.edu.ar

(2) CONICET. Anatomía Comparada, Facultad de Ciencias Naturales y Museo, Universidad Nacional de La Plata. Calle 64 s/n entre Diagonal 113 y 120 (1900) La Plata, Buenos Aires, Argentina. E-mail: fabricioidoeta@hotmail.com 
Espinal y Campos y Malezales (Burkart et al., 1999; Brown et al., 2006). Estas regiones ecológicas tan diversas albergan a su vez una gran riqueza de murciélagos. En el país se encuentran cuatro familias de quirópteros: Phyllostomidae, Noctilionidae, Vespertilionidae y Molossidae, con más de 60 especies. De éstas, más de la mitad se distribuyen en las provincias de Formosa, Chaco, Corrientes y/o Misiones (Barquez y Díaz, 2009).

Los murciélagos por sus características ecológicas particulares se asocian con una fauna de artrópodos en su mayoría específica. Si bien el conocimiento sobre la relación parásito-hospedador, distribución y biología de ectoparásitos (especialmente insectos) se amplió para todo el norte de Argentina, principalmente para las Yungas, son aún escasos los estudios sobre los ectoparásitos en la región Nordeste (Bárquez et al., 1989; Autino, 1989,1996; Autino et al., 1992, 1998, 1999, 2000; Autino y Claps, 2001; Oscherov et al., 2006). El objetivo de este trabajo fue ampliar el conocimiento sobre dípteros ectoparásitos de murciélagos en la provincia de Corrientes.

\section{MATERIALES Y MÉTODOS}

Area de estudio: Los sitios de muestreo incluyeron ambientes silvestres y urbanos. Los murciélagos fueron capturados en tres localidades del Norte de la provincia de Corrientes: Isla Apipé Grande, Puerto Valle y Rincón Santa María.

Hospedadores: La colecta de murciélagos y sus ectoparásitos se realizó durante los meses de diciembre de 2008 a noviembre de 2009. Los hospedadores se obtuvieron por captura manual y con redes de niebla cubriendo una extensión de $42 \mathrm{~m}$, expuestas entre las 19 y las 5 hs de cada día. Los muestreos se realizaron durante tres noches en cada localidad. Los especimenes se colocaron individualmente en bolsas de tela blanca para ser trasladados al laboratorio. Para su identificación se utilizaron claves específicas (Barquez y Díaz, 2009). Los ejemplares de referencia están depositados en el Laboratorio de Anatomía Comparada, Facultad de Ciencias Naturales y Museo, Universidad Nacional de La Plata.

Ectoparásitos: Los murciélagos se examinaron bajo microscopio estereoscópico en busca de los ectoparásitos, los que se colectaron con pinzas entomológicas y conservaron en una solución de alcohol etílico y glicerina. En el laboratorio se procedió identificación, utilizando las claves de Guimarães y D'Andretta (1956), Autino et al. (1999) y Graciolli y de Carvalho (2001). Todos los ejemplares se encuentran depositados en la colección del Laboratorio Biología de los Parásitos, Facultad de Ciencias Exactas y Naturales y Agrimensura, Universidad Nacional del Nordeste.

\section{RESULTADOS}

Las especies que presentaron ectoparásitos fueron Sturnira lilium (Phyllostomidae), Eptesicus furinalis y Myotis nigricans (Vespertilionidae). Las especies de dípteros identificadas fueron Megistopoda proxima (Diptera: Streblidae) Basilia plaumanni y Basilia carteri (Diptera: Nycteribiidae). No se observó la presencia de dos o más 
especies de dípteros en el mismo hospedador. La información detallada se expresa en el siguiente resumen taxonómico:

Familia Nycteribiidae

Basilia carteri Scott, 1936

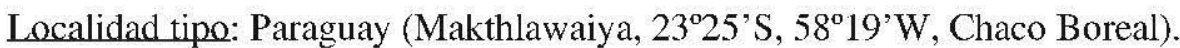

Ejemplares examinados: Argentina. Corrientes, Isla Apipé Grande ( $\left.27^{\circ} 30^{\prime} \mathrm{S}, 56^{\circ} 54^{\prime} \mathrm{W}\right)$ una hembra.

Hospedador: Myotis nigricans (Vespertilionidae).

Prevalencia: $4,2 \%(1 / 24)$

Hospedador tipo: Molossops temminckii (Mollosidae).

Distribución en América del Sur: Argentina, Bolivia, Brasil, Paraguay y Uruguay.

Distribución en la Argentina: Misiones, Corrientes, Chaco, Jujuy, Salta, Santiago del Estero y Tucumán.

Comentarios: Un único ejemplar hembra fue encontrado sobre el hospedador. Basilia carteri fue citada en nuestro país parasitando a $M$. nigricans en Santiago del Estero, Salta y Tucumán (Del Ponte, 1944; García, 1959; Claps et al., 1992). En Corrientes B. carteri fue citada sobre Myotis albescens (Claps et al., 2004) y sobre $M$. nigricans (Oscherov et al., 2006).

\section{Basilia plaumanni Scott, 1940}

Localidad tipo: Santa Catarina, Nova Teutonia, Brasil

Ejemplares examinados: Argentina. Corrientes, Isla Apipé Grande ( $\left.27^{\circ} 30^{\prime} \mathrm{S}, 56^{\circ} 54^{\prime} \mathrm{W}\right)$, dos hembras y dos machos.

Hospedador: Eptesicus furinalis (Vespertilionidae)

Prevalencia: $100 \%(1 / 1)$

Hospedador tipo: Histiotus sp. (Vespertilionidae)

Distribución en América del Sur: Brasil, Paraguay, Uruguay y Argentina.

Distribución en la Argentina: Salta, Jujuy, Santiago del Estero, Córdoba y Corrientes (este trabajo).

Comentarios: Se observaron dos individuos hembras y dos machos en el mismo ejemplar de murciélago. Autino et al. (1999; 2009) colectan este díptero en Histiotus laephotis y también en $E$. furinalis. Otros hospedadores citados en Brasil son $H$. montanus, $H$. macrotus, $H$. velatus y E. fuscus (Graciolli y de Carvalho, 2001) y E. diminutus en Paraguay (Graciolli et al., 2006). Se amplía su distribución a la provincia de Corrientes.

\section{Familia Streblidae}

\section{Megistopoda proxima Sèguy 1926}

Localidad tipo: Argentina, Misiones, inmediaciones de San Ignacio, Villa Lutecia. Ejemplares examinados: Argentina. Corrientes, Ituzaingó, Puerto Valle (273's, $\left.56^{\circ} 23^{\prime} \mathrm{W}\right)$ dos hembras y dos machos. Rincón Santa María (27 $\left.32^{\prime} \mathrm{S}, 56^{\circ} 37^{\prime} \mathrm{W}\right)$ dos hembras. 
Hospedador: Sturnira lilium (Phyllostomidae).

Prevalencia: $25 \%(2 / 8)$

Hospedador tipo: Murciélago no identificado.

Distribución en América del Sur: Argentina, Bolivia, Colombia, Costa Rica, Cuba, Dominica, Guyana Francesa, Islas Vírgenes (St. Croix), Martinica, México, Panamá, Paraguay, Perú, Uruguay y Venezuela.

Distribución en la Argentina: Catamarca, Jujuy, Misiones, Salta, Tucumán y Corrientes (este trabajo).

Comentarios: Fue la especie mas abundante y se hallaron hasta cuatro especimenes en un solo individuo de murciélago. En Argentina fue encontrado en varias especies de Sturnira: S. erythromos, S. lilium y S. oporaphilum (Autino et al., 1992, Autino, 1996). Se cita por primera vez para la provincia de Corrientes.

\section{DISCUSIÓN Y CONCLUSIONES}

El primer registro de $B$. carteri asociada a $M$. nigricans en la provincia de Corrientes correspondió a la eco-región Esteros del Iberá (Oscherov et al., 2006). En este trabajo se amplía su distribución al Delta e Islas del Paraná. Aunque fue recolectada también sobre especies de murciélagos de la familia Molossidae (Molossops temminckii y Tadarida brasiliensis), el reiterado hallazgo sobre $M$. albescens, $M$. riparius, $M$. keaysi y M. nigricans (Autino et al., 1999; Oscherov et al., 2006), indicaría que B. carteri se encuentra en forma habitual en las especies del género Myotis (Claps et al., 1992).

Respecto a B. plaumanni se hace evidente su predilección por los Vespertilionidae (Autino et al., 1999; 2009: Graciolli y de Carvalho, 2001; Graciolli et al., 2006). La frecuencia en que se la colecta sobre murciélagos de los géneros Histiotus y Eptesicus indicaría que ambos serían hospedadores primarios y que la asociación con un hospedador u otro depende de la presencia de los mismos en una región determinada.

$M$. proxima fue colectada en localidades cercanas a Misiones y los registros previos, tanto en Argentina como en otros países de América, señalan que Sturnira sería el hospedador principal (Autino et al., 1992; Autino, 1996; Dick and Gettinger, 2005).

Con el hallazgo de $M$. proxima se aumenta a seis la lista de especies de Streblidae citadas para Corrientes (Autino et al., 2009; este trabajo) y con B. plaumanni son dos las especies de Nycteribiidae presentes en el área de estudio (Oscherov et al., 2006; este trabajo).

\section{AGRADECIMIENTOS}

El presente trabajo contó con el apoyo financiero de la Universidad Nacional del Nordeste (SGCyT), en el marco del proyecto acreditado PI 008/09. 


\section{REFERENCIAS}

AutinO, A.G., 1989. Contribución al conocimiento de la biología de dos especies simpátridas del género Sturnira (Mammalia, Chiroptera). Seminario de Licenciatura Facultad de Ciencias Naturales e Instituto Miguel Lillo-Universidad Nacional de Tucumán, Argentina. 73 p.

AuTIno, A.G.; R.M. BARQuez y G.L. Claps, 1992. Nuevas citas de dípteros ectoparásitos (Streblidae) para murciélagos de la Argentina. Rev. Soc. Entomol. Argentina, 50: 248 y 260.

AutrNo, A.G., 1996. Contribución al conocimiento de la sistemática y biología de los murciélagos de las yungas de la Argentina y sus insectos ectoparásitos. Tesis doctoral, Facultad de Ciencias Naturales e Instituto Miguel Lillo-Universidad Nacional de Tucumán, Argentina. 310 p.

Autino, A.G. and G.L. CLAPS, 2001. Catalogue of the ectoparasitic insects of the bats of Argentina. Insecta Mundi, 14: 193-209.

Autino, A.G.; G.L. Claps y M.P. Bertol.jn, 1998. Primeros registros de insectos ectoparásitos (Diptera, Streblidae) de murciélagos de Parque Nacional Iguazú, Misiones, Argentina. Rev. Bras. Entomol, 42: 59-63.

AuTinO, A.G.; G.L. ClAPS y R.M. BARQuEZ, 1999. Insectos ectoparásitos de murciélagos de las Yungas de la Argentina. Acta Zool. Mex., 78: 119-169.

Autino, A.G.; G.L. Claps y R.M. Barquel, 2000. Nuevos registros de Diptera (Nycteribiidae) y Siphonaptera (Ischnopsyllidae) de Chiroptera (Vespertilionidae) de la Argentina. Bol. Entomol. Venez., 15: 109-112.

AutTino, A.G.; G.L. Ct.APS; M.S. SÁNCHF7. and R.M. BARQue7., 2009. New records of bat ectoparasites (Diptera, Hemiptera and Siphonaptera) from Northern Argentina. Neotrop Entomol., 38: 165-177.

Barquez, R.M.; G.L. Claps and A.G. Autino, 1989. Bat ectoparasitic Diptera from northwestern Argentina. Com. Biol., 8: 157.

BarQuf7, R.M. y M.M. Día7, 2009. Los Murciélagos de Argentina Clave de Identificación (Key to the Bats of Argentina). Publicación Especial N 1 PCMA (Programa de Conservación de los Murciélagos de Argentina), Tucumán.

Burkart, R.; N.O. BArbARo; R.O. SínChez y D.A. Gomez, 1999. Ecorregiones de la Argentina. Administración de Parques Nacionales, Buenos Aires.

BRown, N.A.; U. Martinf7. ORTT7; M. ACFRBI y J. CORCuteRA, 2006. La situación ambiental argentina 2005. Fundación Vida Silvestre, Buenos Aires, Argentina.

ClaPS, G.L.; A.G. Autino y R.M. BARquez, 1992. Nuevas citas de dípteros ectoparásitos (Nycteribiidae) para murciélagos de la Argentina. Rev. Soc. Entomol. Argentina, 50: 88.

Ci.APS, G.L.; A.G. AutTino; M.L. Merino y A.M. ABbA, 2004. Nuevas citas de insectos ectoparásitos de murciélagos para las provincias de Buenos Aires y Corrientes, Argentina. Physis (Buenos Aires), Secc. C., $59(136-137): 53-56$.

Del Ponte, E., 1944. Basilia romañai, nobis (Diptera, Nycteribiidae) de la Argentina. Ann. Inst. Med. Reg. 1: $117-128$.

Dick, C.W. y D. GeTlinger, 2005. A faunal survey of streblid flies (Diptera: Streblidae) associated with bats in Paraguay. J. Parasitol, 91: 1015-1024.

García, M., 1959. Diptera pupipara. Primeras Jornadas entomoepidemiológicas argentinas, 2: 579-580.

Guimaraes, L.R. y M.A.V. D'andretTA, 1956. Sinopse dos Nycteribiidae (Diptera) do Novo Mundo. Arq. Zool. São Paulo. 10: 1-184. 
Gractot t.t, G. y C.J.B. de Carvat.Ho, 2001. Moscas ectoparasitas (Diptera, Hippoboscoidea, Nycteribiidae) de morcegos (Mammalia, Chiroptera) do estado do Paraná, Brasil. I. Basilia, taxonomia e chave pictórica para as espécies. Rev. Bras. Zool, 18: 33-49.

GrACiolli, G.; C.W. DiCK and D. GetTinger, 2006. A faunal survey of nycteribiid flies (Diptera: Nycteribiidae) associated with bats in Paraguay. Zootaxa, 1220: 35-46.

Oscherov, E.B; M.L. Chatellenaz y A.M.F. Milano, 2006. Basilia carteri (Diptera: Nycteribiidae) en murciélagos de la Reserva provincial Iberá (Corrientes, Argentina). Facena, 22: 3-6.

Recibido/Received/: 15-May-2013

Aceptado/Accepted/: 09-Oct-2013 